\title{
AN ASYMPTOTIC FORMULA FOR HYPO-ANALYTIC PSEUDODIFFERENTIAL OPERATORS
}

\author{
S. BERHANU
}

\begin{abstract}
An asymptotic expansion formula for hypo-analytic pseudodifferential operators is proved and applications are given.
\end{abstract}

\section{INTRODUCTION}

In [2] we introduced hypo-analytic pseudodifferential operators that are naturally associated with the hypo-analytic structures of [1]. In this paper we establish an asymptotic formula for these operators. Such an expansion is essential in several applications. It allows us to define, in a natural way, the symbol of a hypo-analytic pseudodifferential operator, as well as the symbols of the adjoint, transpose and composition of operators. The paper is organized as follows. In Chapter I we discuss and develop the asymptotic formula. Chapter II applies this formula to two results.

Acknowledgment. It is a pleasure to express my thanks to Professor F. Treves for many stimulating discussions.

\section{ASYMPTOTIC EXPANSION}

1. Hypo-analytic structures. We will deal with structures which are a special case of the hypo-analytic structures introduced by Baouendi, Chang and Treves in [1]. We shall summarize the relevant concepts here. Let $\Omega$ be a $C^{\infty}$ manifold of dimension $m$. A hypo-analytic structure of maximal dimension on $\Omega$ is the data of an open covering $\left(U_{\alpha}\right)$ of $\Omega$ and for each index $\alpha$, of $m C^{\infty}$ functions $Z_{\alpha}^{1}, \ldots, Z_{\alpha}^{m}$ satisfying the following two conditions:

(i) $d Z_{\alpha}^{1}, \ldots, d Z_{\alpha}^{m}$ are linearly independent at each point of $U_{\alpha}$;

(ii) if $U_{\alpha} \cap U_{\beta} \neq \varnothing$, there are open neighborhoods $\mathscr{O}_{\alpha}$ of $Z_{\alpha}\left(U_{\alpha} \cap U_{\beta}\right)$ and $\mathscr{O}_{\beta}$ of $Z_{\beta}\left(U_{\alpha} \cap U_{\beta}\right)$ and a holomorphic map $F_{\beta}^{\alpha}$ of $\mathscr{O}_{\alpha}$ onto $\mathscr{O}_{\beta}$

such that

$$
Z_{\beta}=F_{\beta}^{\alpha} \circ Z_{\alpha} \text { on } U_{\alpha} \cap U_{\beta} \text {. }
$$

We will use the notation $Z_{\alpha}=\left(Z_{\alpha}^{1}, \ldots, Z_{\alpha}^{m}\right): U_{\alpha} \rightarrow C^{m}$. A distribution $h$ defined in an open neighborhood of a point $p_{0}$ of $\Omega$ is hypo-analytic at $p_{0}$

Received by the editors August 14, 1989.

1980 Mathematics Subject Classification (1985 Revision). Primary 35A20; Secondary 35S99. 
if there is a chart $\left(U_{\alpha}, Z_{\alpha}\right)$ of the above type whose domain contains $p_{0}$ and a holomorphic function $\tilde{h}$ defined on an open neighborhood of $Z_{\alpha}\left(p_{0}\right)$ in $C^{m}$ such that $h=\tilde{h} \circ Z_{\alpha}$ in a neighborhood of $p_{0}$. By a hypo-analytic local chart we mean an $m+1$-tuple $\left(U, Z^{1}, \ldots, Z^{m}\right)$ [abbreviated $(U, Z)$ ] consisting of an open subset $U$ of $\Omega$ and of $m$ hypo-analytic functions whose differentials are linearly independent at every point of $U$.

We will now reason in a hypo-analytic local chart $(U, Z)$ of $\Omega$. Assume that the open set $U$ has been contracted sufficiently so that the mapping $Z=$ $\left(Z^{1}, \ldots, Z^{m}\right): U \rightarrow C^{m}$ is a diffeomorphism of $U$ onto $Z(U)$ and that $U$ is the domain of local coordinates $x_{j}(1 \leq j \leq m)$ all vanishing at a "central point" which will be denoted by 0 . We will suppose $Z(0)=0$ and denote by $Z_{x}$ the Jacobian matrix of the $Z^{j}$ with respect to the $x^{k}$. Substitution of $Z_{x}(0)^{-1} Z(x)$ for $Z(x)$ will allow us to assume that $Z_{x}(0)=$ the identity matrix. Therefore the real part of the $Z^{j}(j=1, \ldots, m)$ can serve as coordinates and in these new coordinates

$$
Z^{j}=x^{j}+\sqrt{-1} \phi^{j}(x), \quad j=1, \ldots, m,
$$

where $\phi=\left(\phi^{1}, \ldots, \phi^{m}\right)$ is real valued with 0 differential at the origin.

Moreover, the functions $Z^{j}$ are selected so that all the derivatives of order two of the $\phi^{j}$ vanish at the origin. Indeed if this is not already so it suffices to replace each $Z^{j}$ by

$$
Z^{j}-\frac{\sqrt{-1}}{2} \sum_{k} \sum_{l} \frac{\partial^{2} \phi^{j}}{\partial x^{k} \partial x^{l}}(0) Z^{k} Z^{l} .
$$

We will use $\check{Z}_{x}$ to denote the transpose of the inverse of the matrix $Z_{x}$. Since the first and second derivatives of all the $\phi^{j}$ are zero at the origin, after contracting $U$ if necessary, we can find a number $c, 0<c<1$ such that for all $x, y$ in $U$ and for all $\xi$ in $R_{m}$

$$
\begin{aligned}
& \left|\Im \check{Z}_{x}(x) \xi\right| \leq c\left|\Re \check{Z}_{x}(x) \xi\right| \quad \text { and } \\
& \Re\left\{\sqrt{-1} \check{Z}_{x}(x) \xi \cdot(Z(x)-Z(y))-\left\langle\check{Z}_{x}(x) \xi\right\rangle(Z(x)-Z(y))^{2}\right\} \\
& \quad \leq-c|\xi||Z(x)-Z(y)|^{2}, \\
& \quad \text { where }\langle\zeta\rangle=\left(\zeta_{1}^{2}+\cdots+\zeta_{m}^{2}\right)^{\frac{1}{2}} \text { for }|\Im \zeta|<|\Re \zeta| .
\end{aligned}
$$

2. Hypo-analytic pseudodifferential operators. We will continue to work in the chart $(U, Z)$ of $\S 1$. Our aim now is to briefly describe the hypo-analytic pseudodifferential operators.

Definition 2.1. Let $d$ be a real number. We denote by $\tilde{S}^{d}(U, U)$ the space of holomorphic functions $\tilde{a}(z, w, \theta)$ in a product set $\mathscr{O} \times \mathscr{O} \times \mathscr{C}$ with $\mathscr{O}$ an open neighborhood of $Z(U)$, and $\mathscr{E}$ an open cone in $C_{m} \backslash\{0\}$ containing $R_{m} \backslash\{0\}$ which have the following property: 
Given any compact subset $K$ of $\mathscr{O}$ and any closed cone $\mathscr{C}^{\prime} \subset \mathscr{C}$ whose interior contains $R_{m} \backslash\{0\}$, there is a constant $r>0$ such that for all $z, w$ in $K$ and all $\theta$ in $\mathscr{C}^{\prime}$, we have

$$
|\tilde{a}(z, w, \theta)| \leq r(1+|\theta|)^{d} .
$$

Definition 2.2. We say that a $C^{\infty}$ function $a(x, y, \theta)$ in $U \times U \times R_{m}$ is a hypo-analytic amplitude of degree $d$ and we write $a \in S^{d}(U, U)$ if there is $\tilde{a} \in \tilde{S}^{d}(U, U)$ such that

$$
a(x, y, \theta)=\tilde{a}(Z(x), Z(y), \theta), \quad \text { for all } x \text { in } U, y \text { in } U, 0 \neq \theta \in R_{m} .
$$

Let $a(x, y, \theta)=\tilde{a}(Z(x), Z(y), \theta)$ be a hypo-analytic amplitude of degree $d \in R$ in $U \times U$. For any $\varepsilon>0$ and $u \in C_{c}^{0}(U)$ we define the linear operator

$$
\begin{array}{r}
A^{\varepsilon} u(x)=\left(\frac{1}{4 \pi^{3}}\right)^{\frac{m}{2}} \int_{U} \int_{R_{m}} \exp \left(\sqrt{-1} \xi \cdot\left(Z(x)-Z(y)-\varepsilon|\xi|^{2}\right)\right. \\
\cdot a(x, y, \xi) u(y) d Z(y) d \xi
\end{array}
$$

We contract $U$ sufficiently so that for every $x, y \in U$ and $\xi \in R_{m}$ the point $\check{Z}_{x}(x) \xi+\sqrt{-1}\left\langle\check{Z}_{x}(x) \xi\right\rangle(Z(x)-Z(y))$ will remain in the cone in which $a(x, y,$.$) is defined. We observe that each A^{\varepsilon} u$ is a hypo-analytic function. The results of [2] may be consolidated into:

Theorem 2.1. When $\varepsilon \rightarrow 0, A^{\varepsilon}$ converges to a continuous linear operator $A$ : $E^{\prime}(U) \rightarrow \mathscr{D}(U)$ which maps $C_{c}^{\infty}(U)$ into $C_{c}^{\infty}(U)$ continuously. If $u$ is hypoanalytic at 0 then $A u$ is hypo-analytic at 0.

The first part of the theorem is proved by first deforming the path of $\xi$ integration from $R_{m}$ to the image of $R_{m}$ under the map

$$
\xi \rightarrow \zeta(\xi)=\check{Z}_{x}(x)(\xi)+\sqrt{-1}\left\langle\check{Z}_{x}(x) \xi\right\rangle(Z(x)-Z(y)) .
$$

The second inequality in (1.1) will then force the exponential term in (2.3) to be bounded. The integral can then be treated as an oscillatory integral.

Following [2] we will call $A$ a hypo-analytic pseudodifferential operator. When $Z(x)=x$ this specializes to the usual analytic pseudodifferential operator.

3. Formal hypo-analytic amplitudes. In this section $(U, Z)$ will be as in $\S 2$. Our aim is to establish an asymptotic expansion formula for hypo-analytic amplitudes.

Fix a neighborhood $\mathscr{O}$ of $Z(U)$ in $C^{m}$, a cone $\mathscr{C}$ in $C^{m} \backslash\{0\}$ and let $R_{0}(z, w)$ be a positive continuous function on $\mathscr{O} \times \mathscr{O}$. For each $j=0,1$, $2, \ldots$ let $k_{j}(z, w, \theta)$ be a holomorphic function in the set

$$
\left\{(z, w, \theta) \in \mathscr{O} \times \mathscr{O} \times \mathscr{C} ;|\theta|>R_{0}(z, w) \sup (j, 1)\right\} .
$$

Set $k_{j}(x, y, \theta)=\tilde{k}_{j}(Z(x), Z(y), \theta)$. 
Definition 3.1. We will say that the series $\sum_{j=0}^{\infty} k_{j}(x, y, \theta)$ defines a formal hypo-analytic amplitude of degree $d$ if there exists a continuous function $c_{0}(z, w)$ $>0$ on $\mathscr{O} \times \mathscr{O}$ such that for all $(z, w)$ in $\mathscr{O} \times \mathscr{O}$ and all $\theta$ in $\mathscr{C},|\theta|>$ $R_{0}(z, w) \sup (j, 1)$,

$$
\left|\tilde{k}_{j}(z, w, \theta)\right| \leq C_{0}(z, w)^{j+1} j !|\theta|^{d-j} .
$$

We now show how to construct a true hypo-analytic amplitude from the formal one given above. We will work in a compact set $K \subseteq U$ and a relatively compact neighborhood $\mathscr{O}_{K}$ of $Z(K)$ in $\mathscr{O}$. This enables us to replace the functions $C_{0}(z, w)$ and $R_{0}(z, w)$ of the above definition by constants $C_{0}$ and $R_{0}$. We will also assume that the cone $\mathscr{C}$ has been shrunk to satisfy: for some $\delta>0$, whenever $\theta=\xi+\sqrt{-1} \eta \in \mathscr{C}$, then $\delta|\theta| \leq|\xi|$. Let $R>\max \left(R_{0}, C_{0}\right)$.

We will use a sequence of smooth cutoff functions $\phi_{j}(\xi)$ having the following properties:

$$
\begin{gathered}
0 \leq \phi_{j}(\xi) \text { for all } \xi, \quad \text { and } \quad \phi_{j}(\xi)=0 \text { in }|\xi|<2 R \sup (j, 1), \\
\phi_{j}(\xi)=1 \quad \text { if }|\xi|>3 R \sup (j, 1) ; \quad\left|D^{\alpha} \phi_{j}\right| \leq\left(\frac{C}{R}\right)^{|\alpha|} \quad \text { if }|\alpha| \leq 2 j .
\end{gathered}
$$

See [8] for the construction of such cutoffs. Define

$$
\tilde{k}(z, w, \theta)=\sum_{j=0}^{\infty} \phi_{j}(\xi) \tilde{k}_{j}(z, w, \theta)
$$

for $(z, w) \in \mathscr{O}_{K} \times \mathscr{O}_{K}$ and $\theta=\xi+\sqrt{-1} \eta \in \mathscr{C} . \quad \tilde{k}$ is a $C^{\infty}$ function of $(z, w, \theta)$ holomorphic in $(z, w) . \tilde{k}$ satisfies the following estimates:

$$
\begin{aligned}
|\tilde{k}(z, w, \theta)| & \leq \sum_{0 \leq j<d}\left|\tilde{k}_{j}(z, w, \theta)\right|+\sum_{j \geq d} \phi_{j}(\xi)\left|k_{j}(z, w, \theta)\right| \\
& \leq \sum_{0 \leq j<d}\left|\tilde{k}_{j}(z, w, \theta)\right|+\sum_{j \geq d} \phi_{j}(\xi) c_{0}^{j+1} j !|\theta|^{d-j} \\
& \leq \sum_{0 \leq j<d}\left|\tilde{k}_{j}(z, w, \theta)\right|+\sum_{j \geq d} \phi_{j}(\xi) c_{0}^{j+1} j !|\xi|^{d-j}
\end{aligned}
$$

Since for $j \geq d$ the $j$ th term lives on the set $\{\xi:|\xi| \geq 2 R j\}$, the latter

$$
\begin{aligned}
& \leq \sum_{0 \leq<j<d}\left|\tilde{k}_{j}(z, w, \theta)\right|+|\xi|^{d} \sum_{j \geq d} c_{0}^{j+1} j !\left(\frac{1}{2 R j}\right)^{j} \\
& \leq \sum_{0 \leq j<d}\left|\tilde{k}_{j}(z, w, \theta)\right|+\text { constant }|\xi|^{d} \\
& \leq \text { constant }|\theta|^{d}
\end{aligned}
$$




$$
\begin{aligned}
\bar{\partial}_{\theta} \tilde{k}(z, w, \theta) & \leq \sum_{j=0}^{\infty}\left|\bar{\partial}_{\theta} \phi_{j}(\xi) \tilde{k}_{j}(z, w, \theta)\right| \\
& \leq\left(\sum_{j=0}^{\infty}\left|\bar{\partial}_{\theta} \phi_{j}(\xi)\right| c_{0}^{j+1} \frac{j !}{|\xi|^{j}}\right) \\
& \leq \delta^{d}|\xi|^{d}\left(\sum_{j=0}^{\infty}\left|\bar{\partial}_{\theta} \phi_{j}(\xi)\right| c_{0}^{j+1} \frac{j !}{|\xi|^{j}}\right)
\end{aligned}
$$

We now use the fact that $\bar{\partial}_{\theta} \phi_{j}(\xi)$ lives in the set $\{\xi: 2 R j \leq|\xi| \leq 3 R j\}$;

$$
\leq \text { constant }|\xi|^{d}\left(\sum_{j=0}^{\infty} c_{0}^{j+1} j !\left(\frac{1}{2 R j}\right)^{j}\right)
$$

Since $j ! / j^{j} \leq e^{-j}$, the latter $\leq$ constant $|\xi|^{d} \sum_{j=0}^{\infty}\left(\frac{c_{0}}{2 R}\right)^{j} e^{-j}$.

Recalling that $2 R j \leq|\xi| \leq 3 R j$, we get

$$
\begin{aligned}
& \leq \text { constant }|\xi|^{d} \sum_{j=0}^{\infty}\left(\frac{c_{0}}{2 R}\right)^{j} e^{-\frac{|\xi|}{3 R}} \\
& \leq \text { constant } e^{-\frac{|\xi|}{4 R}} \\
& \leq \text { constant } e^{-\frac{\delta}{4 R}|\theta|}
\end{aligned}
$$

Thus for $(z, w, \theta) \in \mathscr{O}_{K} \times \mathscr{O}_{K} \times \mathscr{C}$, we have: $|\tilde{k}(z, w, \theta)| \leq$ const. $|\theta|^{d}$ and $\left|\bar{\partial}_{\theta} \tilde{k}(z, w, \theta)\right| \leq$ const. $e^{-\frac{\delta}{2 R}|\theta|}$.

We may assume that the shape of $\mathscr{C}$ has been modified to allow us to solve the Cauchy-Riemann equations in $\mathscr{C}$ (see [5]) $\bar{\partial}_{\theta} \tilde{k}_{1}=\bar{\partial}_{\theta} \tilde{k}$ in such a way that the solution $\tilde{k}_{1}$ is holomorphic with respect to $(z, w)$ in $\mathscr{O}_{K} \times \mathscr{O}_{K}$ and the following estimate holds on sets of the kind $K_{1} \times K_{2} \times \mathscr{C}_{1}\left(K_{1}, K_{2} \subset \subset \mathscr{O}_{K}\right)$ and $\mathscr{C}_{1}$ a cone whose closure is contained in $\mathscr{C}$ :

$$
\left|\tilde{k}_{1}(z, w, \theta)\right| \leq \text { const. } e^{-\frac{\delta}{4 R}|\theta|} .
$$

Define then $h=\tilde{k}-\tilde{k}_{1}$. We now have, in $\mathscr{O}_{K} \times \mathscr{O}_{K} \times \mathscr{C}_{1}, \bar{\partial}_{\theta} \tilde{h}=0$ and $\tilde{k}-\tilde{h}$ decays exponentially as $|\theta| \rightarrow \infty$ (uniformly, provided $(z, w, \theta)$ stays in sets like $K_{1} \times K_{2} \times \mathscr{C}_{1}$ as above).

This decay together with Theorem 2.1 of $\S 2$ imply that if for $u \in \mathscr{E}^{\prime}(U), U$ sufficiently small, we define

$$
\begin{array}{r}
\text { op } \tilde{k}^{\varepsilon} u(x)=\left(\frac{1}{4 \pi^{3}}\right)^{\frac{m}{2}} \int_{U^{\prime}} \int_{R_{m}} e^{\sqrt{-1 \xi} \cdot(Z(x)-Z(y))-\varepsilon|\xi|^{2}} \\
\cdot \tilde{k}(Z(x), Z(y), \xi) u(y) d Z(y) d \xi
\end{array}
$$

then as $\varepsilon \rightarrow 0^{+}$, op $\tilde{k}^{\varepsilon}$ will converge to an operator op $\tilde{k}$ having the properties in Theorem 2.1, §2. Moreover, for any $u \in \mathscr{E}^{\prime}(U)$, op $\tilde{k} u-$ op $\tilde{h} u$ is a hypoanalytic function. We will therefore replace $\hat{k}$ by the hypo-analytic amplitude 
$\tilde{h}$ and think of $\tilde{h}$ as being the true amplitude constructed from the formal one given by $\sum_{j=0}^{\infty} k_{j}(x, y, \theta)$.

4. Asymptotic expansion. Let $k(x, y, \theta)$ be a hypo-analytic amplitude of degree d say $k(x, y, \theta)=\tilde{k}(Z(x), Z(y), \theta)$ where $\tilde{k}$ is holomorphic in $\mathscr{O} \times$ $\mathscr{O} \times \mathscr{C}, \mathscr{O}$ and $\mathscr{C}$ are as in $\S 1$. For each $j=1, \ldots, m$, let $N_{j}$ denote the vector field $N_{j} Z^{k}=-\sqrt{-1} \delta_{j}^{k}$.

If $K \subset U$ is any compact subset, by Cauchy's inequality we have $c>0$ such that:

$$
\left|\frac{1}{\alpha !} \partial_{\xi}^{\alpha} N_{y}^{\alpha} k(x, x, \xi)\right| \leq c^{|\alpha|+1} \alpha !(1+|\xi|)^{d-|\alpha|}
$$

for $x \in K, \breve{\zeta} \in R_{m}$.

Thus if we define

$$
k_{j}(x, \xi)=\sum_{|\alpha|=j} \frac{1}{\alpha !} \partial_{\xi}^{\alpha} N_{y}^{\alpha} k(x, x, \xi)
$$

then $\sum_{j=0}^{\infty} k_{j}(x, \xi)$ can be thought of as a formal hypo-analytic symbol. Let $\left(\phi_{j}\right)_{j}$ be the cutoff functions of the previous section. If $U^{\prime}$ is any relatively compact subset of $U$, we can form a true symbol by setting

$$
k(x, \xi)=\sum_{j=0}^{\infty} k_{j}(x, \xi) \phi_{j}(\xi)
$$

We then have two operators op $k(x, y, \xi)$ and op $\tilde{k}(x, \xi): \mathscr{E}^{\prime}\left(U^{\prime}\right) \rightarrow D^{\prime}\left(U^{\prime}\right)$ where for $u \in \mathscr{E}^{\prime}\left(U^{\prime}\right)$,

$$
\text { op } \tilde{k} u(x)=\lim _{\varepsilon \rightarrow 0^{+}}\left(\frac{1}{4 \pi^{3}}\right)^{\frac{m}{2}} \iint e^{\sqrt{-1}\left(Z(x)-Z(y) \cdot \xi \xi-\varepsilon|\xi|^{2}\right.} k(x, \xi) u(y) d Z(y) d \xi
$$

and

$$
\text { op } k u(x)=\lim _{\varepsilon \rightarrow 0^{+}}\left(\frac{1}{4 \pi^{3}}\right)^{\frac{m}{2}} \iint e^{\sqrt{-1}(Z(x)-Z(y)) \cdot \xi-\varepsilon|\xi|^{2}} k(x, y, \xi) u(y) d Z(y) d \xi
$$

The next theorem proves that if $U^{\prime}$ is small enough, modulo a hypo-analytic regularizing operator, op $k=$ op $\tilde{k}$.

Theorem 4.1. If the neighborhood $U^{\prime}$ is sufficiently small, op $k \equiv$ op $\tilde{k}$ in the sense that for any $u \in \mathscr{D}^{\prime}\left(U^{\prime}\right)$, op $k u-\operatorname{op} \tilde{k}$ is a hypo-analytic function.

Proof. Assume $U^{\prime}$ is an open ball centered at 0 , its size to be determined later. We first take $u \in C_{c}^{0}\left(U^{\prime}\right)$. The theorem will be proved by first establishing:

(i) $($ op $k-\mathrm{op} \tilde{k}) u$ is in $C^{\infty}\left(U^{\prime}\right)$, and

(ii) There exists $c>0$ such that for all $\alpha \in Z_{m}^{+}$,

$$
\begin{aligned}
\left|M^{\alpha}(\operatorname{op} k-\operatorname{op} \tilde{k}) u(x)\right| \leq c^{|\alpha|+1} \alpha ! & \text { where } M_{j}=\sqrt{-1} N_{j} \\
& \text { for each } j=1, \ldots, m .
\end{aligned}
$$


Taylor expansion in $U^{\prime}$ gives

$$
\begin{aligned}
k(x, y, \xi)= & \sum_{|\alpha| \leq N} \frac{(Z(y)-Z(x))^{\alpha}}{\alpha !} M_{y}^{\alpha} k(x, x, \xi) \\
& +\sum_{|\alpha|=N+1}(Z(y)-Z(x))^{\alpha} k_{\alpha}(x, y, \xi)
\end{aligned}
$$

where $k_{\alpha}(x, y, \xi)=(N+1) \int_{0}^{1} M_{y}^{\alpha} k(x, x+t(y-x), \xi)(1-t)^{N} d t$.

For each $N=1,2, \ldots$ we define the amplitudes

$$
\begin{aligned}
& k_{N}(x, y, \xi)=\phi_{N+1}(\xi) k(x, y, \xi), \quad \tilde{k}_{N}(x, y, \xi)=\sum_{j \leq N} \phi_{j}(\xi) k_{j}(x, \xi), \\
& r_{N}(x, \xi)=\sum_{j \leq N}\left(\phi_{N+1}(\xi)-\phi_{j}(\xi)\right) k_{j}(x, \xi), \\
& s_{N}(x, y, \xi)=\left(\sum_{|\alpha|=N+1} \frac{1}{\alpha !} D_{\xi}^{\alpha} k_{\alpha}(x, y, \xi)\right) \phi_{N+1}(\xi), \quad \text { and } \\
& t_{N}(x, y, \xi)=\sum_{|\alpha| \leq N+1} \frac{1}{\alpha !}\left\{D_{\xi}^{\alpha}\left(\phi_{N+1}(\xi) k_{\alpha}(x, y, \xi)\right)-\phi_{N+1}(\xi) D_{\xi}^{\alpha} k_{\alpha}\right\} .
\end{aligned}
$$

Let $K_{N}, \tilde{K}_{N}, R_{N}, S_{N}$ and $T_{N}$ denote the respective operators that are defined in the same fashion as op $k$. We have

$$
(\text { op } k-\text { op } \tilde{k}) u=\left(\tilde{K}_{N}-\text { op } \tilde{k}\right) u+\left(\text { op } k-K_{N}\right) u+R_{N} u+S_{N} u+T_{N} u \text {. }
$$

Our estimates will show that given any positive integer $l$, there exists a positive integer $N$ such that each term on the right-hand side of the above equation is in $C^{\prime}$-thus establishing that $(\operatorname{op} k-\operatorname{op} \tilde{k}) u \in C^{\infty}\left(U^{\prime}\right)$.

(A) Estimate of $M^{\alpha}\left(\right.$ op $\left.k-K_{N}\right) u$. Since the $\xi$-support of

$$
\left(1-\phi_{N+1}(\xi)\right) k(x, y, \xi)
$$

is compact, $\left(\right.$ op $\left.k-K_{N}\right) u$ is hypo-analytic and therefore in particular, $C^{\infty}$.

Suppose $|Z(x)-Z(y)| \leq A$ for all $x, y$ in $U^{\prime}$.

$$
\begin{aligned}
\left|\left(\mathrm{op} k-K_{N}\right) u(x)\right| & =\left(\frac{1}{4 \pi^{3}}\right)^{\frac{m}{2}} \mid \int_{y} \int_{|\xi| \leq 3 R(N+1)} e^{\sqrt{-1}(Z(x)-Z(y)) \cdot \xi} \\
& \cdot k(x, y, \xi)\left(1-\phi_{N}(\xi)\right) d Z(y) d \xi \mid \\
& \leq \text { const. } \int_{|\xi| \leq 3 R(N+1)} e^{A|\xi|}(1+|\xi|)^{d} d \xi
\end{aligned}
$$

(the constant is independent of $N$ )

$\leq$ const. $\left(e^{3 R A}\right)^{N+1}(N+1)^{d+m}$

$\leq c_{1}^{N+1}$ for some $c_{1}>0$ independent of $N$. 
Moreover, since each (op $\left.k-K_{N}\right) u$ is hypo-analytic in a common domain, for example some neighborhood of the compact set $\bar{U}^{\prime}$, we can find a constant $\tilde{c}_{1}>0$ independent of $N$ such that for all $\alpha \in Z_{m}^{+}$,

$$
\mid M^{\alpha}\left(\text { op } k-K_{N}\right) u(x) \mid \leq \tilde{c}_{1}^{|\alpha|+1} c_{1}^{N+1} \alpha !
$$

(B) Estimate of $M^{\alpha}\left(S_{N} u\right)$. Write

$$
s_{N}(x, y, \xi)=\phi_{N+1}(\xi) \sum_{|\alpha|=N+1} D_{\xi}^{\alpha} k_{\alpha}(x, y, \xi)=\phi_{N+1}(\xi) \tilde{s}_{N}(x, y, \xi) .
$$

For $|\alpha|=N+1$, we have

$$
\left|\frac{D_{\xi}^{\alpha} k_{\alpha}(x, y, \xi)}{\alpha !}\right| \leq c^{|\alpha|} \alpha !(1+|\xi|)^{d-N-1} .
$$

It follows that $\left|\tilde{s}_{N}(x, y, \xi)\right| \leq c_{2}^{N+1} N !(1+|\xi|)^{d-N-1}$ for some $c_{2}>0$.

Let

$$
I_{N}^{\varepsilon}(x)=\left(\frac{1}{4 \pi^{3}}\right)^{\frac{m}{2}} \iint e^{\sqrt{-1}(Z(x)-Z(y)) \cdot \xi-\varepsilon|\xi|^{2}} \phi_{N+1}(\xi) \tilde{s}_{N}(x, y, \xi) u(y) d Z(y) d \xi .
$$

We note that $s_{N} u(x)=\lim _{\varepsilon \rightarrow 0^{+}} I_{N}^{\varepsilon}(x)$.

We will deform the path of $\xi$-integration from $R_{m}$ to the image of $R_{m}$ under the map

where

$$
\xi \rightarrow \theta(\xi)=\phi_{2 N}(\xi) \zeta(\xi)+\left(1-\phi_{2 N}(\xi)\right) \xi
$$

$$
\zeta(\xi)=\check{Z}_{x}(x) \xi+\sqrt{-1}\left\langle\check{Z}_{x}(x) \xi\right\rangle(Z(x)-Z(y)) .
$$

The deformation is allowed since it takes place in a region where $\phi_{N+1}(\xi)$ is analytic.

We have

$$
\begin{gathered}
\theta(\xi)= \begin{cases}\xi, & \text { for }|\xi| \leq 4 R N, \\
\zeta(\xi), & \text { for }|\xi| \geq 6 R N .\end{cases} \\
\left|M^{\alpha}\left(I_{N}^{\varepsilon}(x)\right)\right| \leq\left(\frac{1}{4 \pi^{3}}\right)^{\frac{m}{2}} \sum_{\beta<\alpha}\left(\begin{array}{l}
\alpha \\
\beta
\end{array}\right) \mid \iint \xi^{(x-\beta} e^{\sqrt{-1}(Z(x)-Z(y)) \cdot \xi-\varepsilon|\xi|^{2}} \\
\cdot \phi_{N+1}(\xi) M^{\beta} \tilde{s}_{N}(x, y, \xi) u(y) d Z(y) d \xi \mid
\end{gathered}
$$

We use the above contour and pass to the limit to get:

$$
\begin{aligned}
\left|\left(M^{\alpha} s_{N} u\right)(x)\right| \leq & \mid\left(\frac{1}{4 \pi^{3}}\right)^{\frac{m}{2}} \sum_{\beta \leq x}\left(\begin{array}{l}
\alpha \\
\beta
\end{array}\right) \int_{2 R(N+1) \leq|\xi| \leq 6 R N} \int(\theta(\xi))^{\alpha-\beta} \\
& \cdot e^{\sqrt{-1}(Z(x)-Z(y)) \cdot \theta(\xi)} \phi_{N+1}(\xi) M^{\beta} \tilde{s}_{N}(x, y, \xi) u(y) d \theta d Z(y) \\
& +\int_{|\xi| \geq 6 R N} \int(\zeta(\xi))^{(x-\beta} e^{\sqrt{-1}(Z(x)-Z(y)) \cdot \zeta(\xi)} \phi_{N+1}(\xi) \\
& \cdot M^{\beta} \tilde{s}_{N}(x, y, \zeta(\xi)) u(y) d Z(y) d \xi \mid
\end{aligned}
$$


We recall that the exponential in the second integral is bounded $(\S 1,(1.1))$. By hypo-analyticity we get $\tilde{c}_{3}>0$ such that

$$
\forall \beta,\left|M^{\beta} \tilde{s}_{N}(x, y, \xi)\right| \leq c_{3}^{|\beta|+1} \beta ! c_{2}^{N+1} N !(1+|\xi|)^{d-N-1} .
$$

These observations imply that

$$
\begin{aligned}
&\left|M^{\alpha} s_{N} u(x)\right| \leq \text { const } . \sum_{\beta \leq \alpha}\left(\begin{array}{l}
\alpha \\
\beta
\end{array}\right) \int_{2 R(N+1) \leq|\xi| \leq 6 R N}|\xi|^{\alpha-\beta} e^{A|\xi|} c_{3}^{N+|\beta|+2} \\
& \cdot \beta ! N !(1+|\xi|)^{d-N-1} d \xi+\sum_{\beta \leq \alpha}\left(\begin{array}{l}
\alpha \\
\beta
\end{array}\right) \\
&\left.\cdot \int_{6 R N \leq|\xi|}|\xi|^{\alpha-\beta} c_{3}^{N+|\beta|+2} \beta ! N !(1+|\xi|)^{d-N-1} d \xi\right)
\end{aligned}
$$

for some $c_{3} \geq \max \left(\tilde{c}_{3}, c_{2}\right)$. Hence, after modifying $c_{3}$ if necessary, we get

$$
\begin{aligned}
\left|M^{\alpha} s_{N} u(x)\right| & \leq \alpha !\left(\sum_{\beta \leq \alpha} \frac{1}{(\alpha-\beta) !} \int_{2 R(N+1) \leq|\xi|}(1+|\xi|)^{|\alpha-\beta|+d-N-1} N ! d \xi\right) c_{3}^{N} \\
& \leq \alpha ! c_{3}^{N}\left(\sum_{\beta \leq \alpha} \frac{1}{(\alpha-\beta) !}\left(\frac{1}{1+2 R N}\right)^{N-|\alpha-\beta|-d-m+1} N !\right) \\
& \leq \alpha ! c_{3}^{N}\left(\sum_{\beta \leq \alpha} \frac{1}{(\alpha-\beta) !}(1+2 R N)^{|\alpha-\beta|+d+m-1}\right)\left(\frac{1}{2 R}\right)^{N} \frac{N !}{N^{N}} \\
& \leq \alpha ! c_{3}^{N}\left(\sum_{\beta \leq \alpha} \frac{1}{(\alpha-\beta) !}(1+2 R N)^{|\alpha-\beta|+d+m-1}\right)\left(\frac{1}{2 R e}\right)^{N} N e \\
& \leq \alpha ! c_{3}^{N}\left(\sum_{\beta \leq \alpha} \frac{1}{(\alpha-\beta) !}(|\alpha-\beta|+d+m-1) ! e^{1+2 R N}\right)\left(\frac{1}{2 R e}\right)^{N} N e \\
\therefore & \leq \alpha ! c_{3}^{N}\left(\sum_{\beta \leq \alpha} \frac{1}{(\alpha-\beta) !}(|\alpha-\beta|+d+m-1) !\right)\left(\frac{e^{2 R}}{2 R e}\right)^{N} N e^{2} .
\end{aligned}
$$

Using the inequality: $(k+l) ! \leq 2^{k+l} k ! l !$ for any positive integers $k$ and $l$, the latter is dominated by

$$
\alpha ! c_{3}^{N}\left(\sum_{\beta \leq \alpha} \frac{1}{(\alpha-\beta) !}|\alpha-\beta| !\right) 2^{|\alpha|+d+m-1}\left(\frac{e^{2 R}}{2 R e}\right)^{N} N e^{2} .
$$

For $|\alpha| \leq N$, we can find another constant which we will still call $c_{3}$ such that the above quantity $\leq \alpha ! c_{3}^{N}$. 
(C) Estimate of $M^{\alpha}\left(\right.$ op $\left.\tilde{k}-\tilde{K}_{N}\right) u$. Let

$$
\begin{aligned}
& J^{\varepsilon} u(x)=\left(\frac{1}{4 \pi^{3}}\right)^{\frac{m}{2}} \iint e^{\sqrt{-1}(Z(x)-Z(y)) \cdot \xi-\varepsilon|\xi|^{2}} \\
& \cdot\left(\sum_{j>N} \phi_{j}(\xi) k_{j}(x, \xi)\right) u(y) d Z(y) d \xi .
\end{aligned}
$$

For each $j>N$, we will use the contour

$$
\theta_{j}(\xi)=\phi_{2 j}(\xi) \zeta(\xi)+\left(1-\phi_{2 j}(\xi)\right) \xi= \begin{cases}\xi, & \text { when }|\xi| \leq 4 R j \\ \zeta(\xi), & \text { when }|\xi| \geq 6 R j .\end{cases}
$$

In the quantity

$$
\begin{aligned}
M^{\alpha}\left(J^{\varepsilon} u\right)(x)=\left(\frac{1}{4 \pi^{3}}\right)^{\frac{m}{2}} \sum_{j>N}\left[\sum_{\beta \leq \alpha}\left(\begin{array}{l}
\alpha \\
\beta
\end{array}\right) \iint \xi^{(\alpha-\beta} e^{\sqrt{-1}(Z(x)-Z(y)) \cdot \xi-\varepsilon|\xi|^{2}}\right. \\
\left.\cdot \phi_{j}(\xi) M^{\beta} k_{j}(x, \xi) \mathcal{u}^{\prime}(y) d Z d \xi\right]
\end{aligned}
$$

we use the contours $\theta^{j}$ in each term of the sum and take limits to get

$$
M^{\alpha}\left(\operatorname{op} \tilde{k}-\tilde{K}_{N}\right) u(x)=\sum_{j>N}\left(I_{1}^{j}(x)+I_{2}^{j}(x)\right)
$$

where

$$
\begin{aligned}
& I_{1}^{j}(x)=\left(\frac{1}{4 \pi^{3}}\right)^{\frac{m}{2}} \sum_{\beta \leq \alpha}\left(\begin{array}{l}
\alpha \\
\beta
\end{array}\right) \int_{2 R j \leq|\xi| \leq 6 R j} \int \theta^{j}(\xi) e^{\sqrt{-1}(Z(x)-Z(y)) \cdot \theta^{j}(\xi)} \\
& \cdot \phi_{j}(\xi) M^{\beta} k_{j}\left(x, \theta^{j}(\xi)\right) u(y) d Z d \theta^{j}
\end{aligned}
$$

while $I_{2}^{j}(x)$ is a similiar expression except that the integration in $\xi$ is carried out over the region $\{\xi:|\xi| \geq 6 R j\}$.

Assuming that $|\alpha| \leq N-d-m$, we have

$$
\begin{aligned}
\left|I_{1}^{j}(x)\right| & \leq \text { const. } \sum_{\beta \leq \alpha}\left(\begin{array}{c}
\alpha \\
\beta
\end{array}\right) \int_{2 R j \leq|\xi| \leq 6 R j}(1+|\xi|)^{d-j+|\alpha|-|\beta|}\left(e^{6 R A}\right)^{j} c_{0}^{|\beta|+j+1} j ! \beta ! d \xi \\
& \leq \text { const } .\left(c_{0} e^{6 R A}\right)^{j} \alpha !\left|\sum_{\beta \leq \alpha} \frac{1}{(\alpha-\beta) !} \int_{2 R j \leq|\xi| \leq 6 R j}(1+|\xi|)^{d-j+|\alpha|-|\beta|} j !\right| c_{0}^{N} \\
& \leq \text { const } .\left(c_{0} e^{6 R A}\right)^{j} \alpha !\left|\sum_{\beta \leq \alpha} \frac{1}{(\alpha-\beta) !} \int_{0 \leq \rho \leq 6 R j} \rho^{d-j+|\alpha|-|\beta|+m-1} j ! d \rho\right| c_{0}^{N}
\end{aligned}
$$

(We have used the fact that $d-j+|\alpha| \leq 0$.)

$$
\leq \text { const. } \alpha !\left(\frac{c_{0} e^{6 R A}}{6 R}\right)^{j}(6 R)^{d+m+N} c_{0}^{N}\left(\sum_{\beta \leq r} \frac{1}{(\alpha-\beta) !} \frac{j !}{j^{j-d-m-|\alpha|+|\beta|}}\right) .
$$


Therefore, for some $\tilde{c}_{4}>0$ independent of $j$ and $N$,

$$
\left|I_{1}^{j}(x)\right| \leq \alpha ! c_{4}^{N+1}\left(\frac{c_{0} e^{6 R A}}{6 R}\right)^{j}
$$

Similarly, after modifying the constant $\tilde{c}_{4}$ if necessary,

$$
\begin{aligned}
\left|I_{2}^{j}(x)\right| & \leq \text { const. } \alpha ! \sum_{\beta \leq \alpha} \frac{1}{(\alpha-\beta) !}\left(\frac{1}{1+6 R j}\right)^{j-d-m-|\alpha|+|\beta|} c_{0}^{|\beta|+j+1} j ! \\
& \leq \alpha ! c_{4}^{N+1}\left(\frac{c_{0}}{6 R}\right)^{j}
\end{aligned}
$$

We recall that $c_{0} \leq R$. At this point we choose $U^{\prime}$ so small that if $A=$ $\sup _{x, y \in U^{\prime}}|Z(x)-Z(y)|$, then $c_{0} e^{6 R A}<6 R$.

We then get a constant $c_{4}>0$ such that: $\mid M^{\alpha}\left(\right.$ op $\left.\tilde{k}-\tilde{K}_{N}\right) u(x) \mid \leq \alpha ! c_{4}^{N+1}$ for $|\alpha| \leq N-d-m$.

(D) Estimate of $M^{\alpha}\left(R_{N} u\right)$.

$$
\begin{aligned}
R_{N} u(x)=\left(\frac{1}{4 \pi^{3}}\right)^{\frac{m}{2}} \iint e^{\sqrt{-1}(Z(x)-Z(y)) \cdot \xi} u(y) \\
\cdot\left(\sum_{j \leq N}\left(\phi_{N+1}(\xi)-\phi_{j}(\xi)\right) k_{j}(x, \xi)\right) d Z(y) d \xi
\end{aligned}
$$

is hypo-analytic since each $\phi_{N+1}-\phi_{j}$ is supported in $2 R j \leq|\xi| \leq 3 R(N+1)$.

We estimate

$$
\begin{gathered}
\left|\sum_{j \leq N}\left(\phi_{N+1}(\xi)-\phi_{j}(\xi)\right) k_{j}(x, \xi)\right| \leq\left(\sum_{j \leq N} c_{0}^{j+1} j !|\xi|^{-j}\right)|\xi|^{d} \\
\quad \leq\left(\sum_{j \leq N} c_{0}^{j+1} j !\left(\frac{1}{2 R j}\right)^{j}\right)|\xi|^{d} \quad(\text { since } 2 R j \leq|\xi|) \\
\leq\left(\sum_{j \leq N}\left(\frac{c_{0}}{2 R e}\right)^{j} j\right) c_{0} e|\xi|^{d} \quad \text { since } \frac{j !}{j^{j}} \leq j e^{-j+1} .
\end{gathered}
$$

It follows that

$$
\left|R_{N} u(x)\right| \leq \text { constant } \int_{|\xi| \leq 3 R(N+1)}|\xi|^{d} d \xi \leq \text { const } .3 R(N+1)^{d+m}
$$

which in turn implies that there is a constant $\tilde{c}_{5}>0$ such that $\left|R_{N} u(x)\right| \leq$ $\tilde{c}_{5}^{N+1}$. Moreover, by hypo-analyticity, we get $c_{5}>0$ satisfying $\left|M^{\alpha} R_{N} u(x)\right| \leq$ $\alpha ! c_{5}^{N+1}$ for all $|\alpha| \leq N$.

(E) Estimate of $M^{\alpha}\left(T_{N} u\right)$.

$$
T_{N} u(x)=\lim _{\varepsilon \rightarrow 0}\left(\frac{1}{4 \pi^{3}}\right)^{\frac{m}{2}} \iint e^{\sqrt{-1}(Z(x)-Z(y)) \cdot \xi-\varepsilon|\xi|^{2}} t_{N}(x, y, \xi) u(y) d Z(y) d \xi
$$


where

$$
t_{N}(x, y, \xi)=\sum_{|\alpha| \leq N+1} \frac{1}{\alpha !}\left\{\left(D_{\xi}^{\alpha}\left(\phi_{N+1}(\xi) k_{\alpha}(x, y, \xi)\right)-\phi_{N+1}(\xi) D_{\xi}^{\alpha} k_{\alpha}(x, y, \xi)\right\} .\right.
$$

We can therefore take the limit under the integral sign and write

$$
T_{N} u(x)=\sum_{|\alpha| \leq N+1} A_{\alpha}(x)
$$

where for each $\alpha,|\alpha| \leq N+1$,

$$
\begin{array}{r}
A_{\alpha}(x)=\left(\frac{1}{4 \pi^{3}}\right)^{\frac{m}{2}} \sum_{0 \neq \beta \leq N} \int_{2 R(N+1) \leq|\xi| \leq 3 R(N+1)} \int e^{\sqrt{-1} Z((x)-Z(y)) \cdot \xi} \frac{1}{\beta !} \\
\cdot\left(D_{\xi}^{\beta} \phi_{N+1}(\xi)\right) \frac{D_{\xi}^{\alpha-\beta} k_{\alpha}(x, y, \xi)}{(\alpha-\beta) !} u(y) d Z(y) d \xi
\end{array}
$$

Therefore

$$
\begin{aligned}
\left|A_{\alpha}(x)\right| \leq & \text { const. } \alpha ! c_{0}^{|\alpha|+1}\left(e^{3 R A}\right)^{N+1}\left[\sum_{0 \neq \beta \leq \alpha} \frac{1}{\beta !}\left(\frac{[3 R(N+1)]^{d+m+1}}{[2 R(N+1)]^{|\alpha-\beta|}}\right)\left(\frac{c_{0}}{R}\right)^{|\beta|}\right] \\
\leq & \text { const } \cdot \frac{\alpha !}{[2 R(N+1)]^{|\alpha|}}\left(e^{3 R A}\right)^{N+1} c_{0}^{|\alpha|+1} \\
& \cdot[3 R(N+1)]^{d+m+1}\left(\sum_{0 \leq \beta \leq \alpha} \frac{\left[2(N+1) c_{0}\right]^{|\beta|}}{\beta !}\right) .
\end{aligned}
$$

Since $|\alpha| \leq N$ and $R$ may be taken to be larger than 1, we know that the factor $\frac{\alpha !}{[2 R(N+1)]^{[a t}} \leq 1$. Therefore, we conclude that there is a constant $c_{6} \geq 0$ for which $\left|M^{\alpha}\left(T_{N} u\right)\right| \leq c_{6}^{N+1} N$ ! whenever $|\alpha| \leq N$.

From (a)-(e) we conclude that there is a positive number $c$ such that

$$
\mid M^{\alpha}(\text { op } k-\text { op } \tilde{k}) u(x) \mid \leq c^{N+1} N \text { ! }
$$

for all $\alpha,|\alpha| \leq N-m-d$.

If we take $|\alpha|=N-m-d$, we can get a constant $\tilde{c} \geq c$ satisfying:

$$
\forall \alpha, \mid M^{\alpha}(\text { op } k-\text { op } \tilde{k}) u(x) \mid \leq \tilde{c}^{|\alpha|+1} \alpha ! \text { for every } x \in U^{\prime} .
$$

By using integration by parts we also reach the same conclusion for $u \in$ $\mathscr{E}^{\prime}\left(U^{\prime}\right)$. Indeed all we need is a representation of the form $u=\sum_{|\alpha| \leq N} M^{\alpha} u_{\alpha x}$ where each $u_{\alpha} \in C_{c}^{0}\left(U^{\prime}\right)$ which is always possible. We have thus shown that (op $k-$ op $\tilde{k}) u$ is in $C^{\infty}\left(U^{\prime}\right)$ and that there is $c>0$ such that for all $\alpha \in Z_{m}^{+}$,

$$
\mid M^{\alpha}(\text { op } k-\text { op } \tilde{k}) u(x) \mid \leq c^{|\alpha|+1} \alpha ! \text {. }
$$

By Theorem 3.1 of [1] it follows that op $k u-$ op $\tilde{k} u$ is a hypo-analytic function. 


\section{Applications}

1. Parametrix for an elliptic operator. As an application of Theorem 4.1 we consider here the construction of a parametrix for an elliptic hypo-analytic differential operator. We will begin by composing a hypo-analytic differential operator $A$ with a hypo-analytic pseudodifferential operator $B$. In [3] we introduced hypo-analytic differential operators. In the local chart $(U, Z)$, the operator $A$ is given by $A=\sum_{|\alpha| \leq n} a_{\alpha}(x) N^{\alpha}$ where each $a_{\alpha}(x)$ is a hypo-analytic function and $N_{j}=-\sqrt{-1} M_{j}$ for $j=1, \ldots, m$.

Theorem 4.1 of the previous chapter allows us to represent the operator $B$ by a symbol $b(x, \theta)$. From $\S 2$, Theorem 2.1 we know that both $B \circ A$ and $A \circ B$ are continuous linear maps from $\mathscr{E}^{\prime}(U)$ to $\mathscr{D}^{\prime}(U)$. We first assume that the operator $A=a(x) N^{\beta}$ for some hypo-analytic function $a(x)$ and some index $\beta$. Then $B(A u)(x)$ is by definition the limit as $\varepsilon \rightarrow+0$ of

$$
B^{\varepsilon}(A u)(x)=\left(\frac{1}{4 \pi^{3}}\right)^{\frac{m}{2}} \iint e^{\sqrt{-1}(Z(x)-Z(y)) \cdot \xi-\varepsilon|\xi|^{2}} b(x, \xi) a(y) N^{\beta} u(y) d Z(y) d \xi .
$$

On the other hand, $\lim _{\varepsilon \rightarrow 0^{+}} B^{\varepsilon}(A u)(x)=C \circ\left(N^{\beta} u\right)(x)$ where $C$ is a hypoanalytic pseudodifferential operator with amplitude given by $b(x, \xi) a(y)$. Therefore, Theorem 4.1 tells us that $C$ can be represented by the symbol $c(x, \xi)=\sum_{\alpha} \frac{\partial_{\xi}^{\prime \prime} b N^{\prime \prime} a(x)}{\alpha !}$. It follows that modulo a hypo-analytic function, we can write

$$
B(A u)(x)=\lim _{\varepsilon \rightarrow 0^{+}}\left(\frac{1}{4 \pi^{3}}\right)^{\frac{m}{2}} \iint e^{\sqrt{-1}(Z(x)-Z(y)) \cdot \xi-\varepsilon|\xi|^{2}} \xi^{\beta} c(x, \xi) u(y) d Z(y) d \xi .
$$

The latter says that a symbol of $B \circ A$ is given by

$$
\xi^{\beta} c(x, \xi)=\sum_{\alpha} \frac{\partial_{\xi}^{\alpha} b(x, \xi) N^{\alpha}\left(a(x) \xi^{\beta}\right)}{\alpha !} .
$$

On the other hand, applying the operator $A$ to

$$
B^{\varepsilon} u(x)=\left(\frac{1}{4 \pi^{3}}\right)^{\frac{m}{2}} \iint e^{\sqrt{-1}(Z(x)-Z(y)) \cdot \xi-\varepsilon|\xi|^{2}} b(x, \xi) u(y) d Z(y) d \xi
$$

gives

$$
\begin{array}{r}
A\left(B^{\varepsilon} u(x)\right)=\left(\frac{1}{4 \pi^{3}}\right)^{\frac{m}{2}} \iint e^{\sqrt{-1}(Z(x)-Z(y)) \cdot \xi-\varepsilon|\xi|^{2}} \\
\cdot\left(\sum_{y \leq \beta}\left(\begin{array}{l}
\beta \\
y
\end{array}\right) \xi^{\beta-\gamma} a(x) N^{\beta} b(x, \xi)\right) u(y) d Z d \xi \\
=\left(\frac{1}{4 \pi^{3}}\right)^{\frac{m}{2}} \iint e^{\sqrt{-1}\left(Z(x)-\left(Z(y) \cdot \xi-\varepsilon|\xi|^{2}\right.\right.} \\
\cdot\left(\sum_{\alpha}\left[\frac{\partial_{\xi}^{\alpha}\left(a(x) \xi^{\beta}\right) N^{\alpha} b(x, \xi)}{\alpha !}\right]\right) u(y) d Z d \xi .
\end{array}
$$


This means that $A \circ B$ has a symbol given by

$$
\sum_{\alpha} \frac{\partial_{\xi}^{\alpha}\left(a(x) \xi^{\beta}\right) N^{\alpha} b(x, \xi)}{\alpha !} \text {. }
$$

By linearity, we will have the same formulas for the symbol of $B \circ A$ and $A \circ B$ when $\mathrm{A}$ is also given by $A=\sum_{|\alpha| \leq n} a_{\alpha}(x) N^{\alpha}$.

We have thus shown that if either $A$ or $B$ is hypo-analytic differential operator, the composition $A \circ B$ is hypo-analytic pseudodifferential operator with symbol

$$
\sum_{\alpha} \frac{\partial_{\xi}^{\alpha} a(x, \xi) N^{\alpha} b(x, \xi)}{\alpha !} .
$$

Definition 1.1. Let $P=\sum_{|\alpha| \leq k} a_{c \gamma}(Z(x)) M^{\prime \alpha}$ where the $a_{c \mid}(z)$ are holomorphic in a neighborhood of $Z(U)$ in $C^{m}$. We say a point $(x, \xi) \in T^{*} U \backslash\{0\}$ is in the characteristic set of $P$ if the point $\left(Z(x), \check{Z}_{x}(x) \xi\right)$ is in the characteristic set of $P^{Z}=\sum_{|\alpha| \leq k} a_{\alpha}(z)\left(\frac{\partial}{\partial z}\right)^{\alpha}$.

Notation. Char $P=$ the characteristic set of $P$ as given by Definition 1.1.

Definition 1.2. A hypo-analytic differential operator $P$ is said to be elliptic at a point $x \in U$ if for every $(x, \xi) \in T^{*} U,(x, \xi) \notin \operatorname{Char} P$.

Now suppose $P=\sum_{|\alpha| \leq k} a_{\alpha}(Z(x)) M^{\alpha}$ is a hypo-analytic differential operator that is elliptic at our central point $0 \in U$. Since $Z(0)=0$ and $d Z(0)=$ Id, we can find a neighborhood $\mathscr{O}$ of 0 in $C^{m}$, a cone $\mathscr{C}$ in $C_{m}$ containing $R_{m} \backslash\{0\}$ and constants $c, R>0$ such that: when $z \in \mathscr{O}$ and $\zeta \in \mathscr{C},|\zeta| \geq R$ we have $\left|\sum_{|\alpha| \leq k} a_{x}(z) \zeta^{\alpha}\right| \geq c|\zeta|^{k}$.

We now have all the ingredients we need to state

Theorem 1.1. Let $A$ be hypo-analytic differential operator in $\Omega$ that is elliptic of order $d$. Given any relatively compact open subset $\tilde{\Omega}$ of $\Omega$, there is a hypoanalytic pseudodifferential operator $B$ in $\Omega$ of order $-d$ such that $A B-I$ and $B A-I$ are hypo-analytic regularizing in $\tilde{\Omega}$.

The proof of this theorem is a simple adaptation of that of the corresponding theorem for analytic pseudodifferential operators as given by Treves [8]. Therefore we omit it.

2. Propagation of hypo-analyticity. In [3] it was shown that hypo-analytic singularities for solutions propagate along the bicharacteristics of hypo-analytic differential operators. Here we extend this result to what may be called classical hypo-analytic pseudodifferential operator. This result may also be viewed as an extension of a theorem of Hanges [4].

We will work in the hypo-analytic local chart $(U, Z)$ of Chapter I. Let $P$ be a classical hypo-analytic pseudodifferential operator with principal symbol $p$. Let $t \rightarrow(x(t), \xi(t))=\gamma(t)$ be a curve in $T^{*} U \backslash\{0\}$ and set $\tilde{\gamma}(t)=(\tilde{x}(t), \tilde{\xi}(t))=$ $\left(Z(x(t)), \check{Z}_{x}(x(t)) \xi(t)\right)$. 
Definition 2.1. The curve $\gamma(t)$ is said to be a bicharacteristic for $P$ if the equations

$$
\frac{d \tilde{x}}{d t}=\frac{\partial p}{\partial \xi}(\tilde{x}(t), \tilde{x}(x)), \quad \frac{d \tilde{\xi}}{d t}=\frac{-\partial p}{\partial z}(\tilde{x}(t), \tilde{\xi}(t))
$$

hold.

We can now state the theorem of this section.

Theorem 2.1. Assume $p\left(0, \xi_{0}\right)=0$ and $P$ is of principal type at $\left(0, \xi_{0}\right)$. Suppose $\gamma=\{(x(t), \xi(t))\}$ is a bicharacteristic for $P$ through $(x(0), \xi(0))=\left(0, \xi_{0}\right)$ and that $P u$ is hypo-analytic on $\gamma$. Then either $u$ is hypo-analytic at every point of $\gamma$ or $u$ is not hypo-analytic at any point of $\gamma$.

The proof will use a version of the FBI transform as developed by Sjöstrand in [7]. We will therefore first discuss Sjöstrand's FBI transformations adapted to our situation here.

Let $H$ be a totally real submanifold of $C^{m}$ of maximal dimension with defining functions $h_{1}, \ldots, h_{m}$.

Define

$$
\Lambda_{H}=\left\{\left(x, \frac{2}{i} \partial h(x)\right): h \in C^{\infty}\left(C^{m}, R\right), h \equiv 0 \text { on } H\right\} .
$$

Note that if $x_{0} \in H$, then $\left(x_{0}, \xi_{0}\right) \in \Lambda_{H}$ iff $\exists$ real numbers $t_{1}, \ldots, t_{m} \ni$

$$
\xi_{0}=\frac{2}{i} \sum_{j=1}^{m} t_{j} \partial h_{j}\left(x_{0}\right) .
$$

Fix a point $\left(y_{0}, \eta_{0}\right) \in \Lambda_{H}$. Let $\varphi$ be a holomorphic function defined near $\left(x_{0}, y_{0}\right) \ni$

(2.1) $\frac{\partial \varphi}{\partial y}\left(x_{0}, y_{0}\right)=-\eta_{0}$,

(2.2) $\operatorname{det} \frac{\partial^{2} \varphi}{\partial x \partial y}\left(x_{0}, y_{0}\right) \neq 0$,

(2.3) $\left.\Im \varphi_{y y}\left(x_{0}, y_{0}\right)\right|_{T_{y_{0}} H \times T_{y_{0}} H}>0$.

Here $\Im \varphi$ is considered as a function on $C^{n} \times H$, defined locally.

Set

$$
\varphi_{1}(x, y)=-\Im \varphi(x, y) .
$$

Condition (2.1) implies that $H \ni y \mapsto \varphi_{1}\left(x_{0}, y\right)$ has a critical point at $y_{0}$ since $\frac{2}{i} \frac{\partial \varphi_{1}}{\partial y}\left(x_{0}, y_{0}\right)=\frac{\partial \varphi}{\partial y}\left(x_{0}, y_{0}\right)=-\eta_{0}$ and that therefore $d_{y} \varphi_{1}\left(x_{0}, y_{0}\right)=$ $d h\left(y_{0}\right)$ for some $h$ vanishing on $H$. This together with condition (2.3) and the implicit function theorem give us neighborhoods $N\left(x_{0}\right)$ of $x_{0}$ in $C^{m}, N\left(y_{0}\right)$ of $y_{0}$ in $H$ and a unique $C^{\infty}$ function $y=y(x): N\left(x_{0}\right) \rightarrow N\left(y_{0}\right)$ such that $y(x)$ is the unique critical point for $H \ni y \mapsto \varphi_{1}(x, y), x \in N\left(x_{0}\right)$. We next note that for $x \in N\left(x_{0}\right),\left(y(x), \frac{-2}{i} \frac{\partial \varphi_{1}}{\partial y}(x, y(x))\right) \in \Lambda_{H}$. Indeed, this follows from the fact that $H \ni y \mapsto \varphi_{1}(x, y)$ has a critical point at $y(x)$ and that $h_{1}, \ldots, h_{m}$ are the defining functions for $H$. 
For $x \in N\left(x_{0}\right)$, let $\eta(x)=\frac{-2}{i} \frac{\partial \varphi_{1}}{\partial y}(x, y(x))$. Then

$$
(y(x), \eta(x))=\left(y(x), \frac{-2}{i} \frac{\partial \varphi_{1}}{\partial y}(x, y(x))\right) \in \Lambda_{H} .
$$

Moreover, for $x$ in $N\left(x_{0}\right), y(x)$ is the unique point in $N\left(y_{0}\right)$ such that

$$
\frac{-\partial \varphi}{\partial y}(x, y(x))=\frac{-2}{i} \frac{\partial \varphi_{1}}{\partial y}(x, y(x)) \in\left(\Lambda_{H}\right)_{y(x)} .
$$

This is due to the uniqueness of the critical point.

Let $\Phi(x)=\varphi_{1}(x, y(x))$. Let $a(x, y, \lambda)$ be a classical analytic symbol defined near $\left(x_{0}, y_{0}\right)$ and elliptic at this point. For $\Psi$ a real-valued function defined on an open set $W$ in $C^{m}$, we define the space $H_{\Psi}^{\text {loc }}(W)=\{v$ : $W \times R_{+} \rightarrow C: v(z, \lambda)$ is holomorphic in $z$ and for any $K \subset \subset W$ and $\varepsilon>$ $0 \exists c \ni|v(z, \lambda)| \leq c e^{\lambda(\psi(z)+\varepsilon)}$ for all $\left.z \in K, \lambda \geq 1\right\}$.

Let $u \in D^{\prime}\left(N\left(y_{0}\right)\right)$, and for $z$ in $N\left(x_{0}\right)$ set

$$
T u(z, \lambda)=\int_{H} e^{i \lambda \varphi\left(z, y^{\prime}\right)} a(z, y, \lambda) \chi(y) u(y) d y
$$

where $\chi \in C_{0}^{\infty}\left(N\left(y_{0}\right)\right), \chi \equiv 1$ near $y_{0}$.

Here we are assuming that the neighborhoods $N\left(y_{0}\right)$ and $N\left(x_{0}\right)$ have been contracted so that the symbol $a$ and the phase function $\varphi$ are defined. It is easily checked that

$$
T: D^{\prime}\left(N\left(y_{0}\right)\right) \longrightarrow H_{\Phi}^{\text {loc }}\left(N\left(x_{0}\right)\right) .
$$

In the sequel, $W F_{h a} u$ denotes the hypo-analytic wave front set of BaouendiChang-Treves [1]. Our proof of Theorem 2.1 will use the following proposition of Sjöstrand [7].

Proposition 2.1. Let $z_{1} \in N\left(y_{0}\right)$. Then $\left(y\left(z_{1}\right), \eta\left(z_{1}\right)\right) \notin W F_{h a} u$ iff $T u \in$ $H_{\Phi-\varepsilon_{0}}^{\text {loc }}(W)$ for some $\varepsilon_{0}>0$ and some neighborhood $W$ of $z_{1}$.

Proof of Theorem 2.1. In order to obtain a suitable phase function, we will need the following two lemmas from [6]. For notational convenience we will use $y_{0}$ for $0 \in Z(U)=H$.

Lemma 2.1. Set $z_{0}=\left(y_{0}^{\prime}-i \xi_{0}^{\prime}, 0\right) \in C^{n-1} \times C$. There exists a holomorphic function $\varphi$ defined near $\left(z_{0}, y_{0}\right)$ which solves

$$
\frac{\partial \varphi}{\partial z_{n}}(z, y)=p\left(y, \frac{-\partial \varphi}{\partial y}(z, y)\right)
$$

and satisfies (2.1)-(2.3) with $\eta_{0}=\xi_{0}$.

We remark that the lemma is proved by using the Cauchy-Kovalevska theorem, which guarantees the existence of a holomorphic $\varphi$ that solves the initial value problem

$$
\frac{\partial \varphi}{\partial z_{n}}=p\left(y, \frac{-\partial \varphi}{\partial y}\right)
$$


and

$$
\varphi(z, 0, y)=\frac{i}{2} \sum_{j=1}^{n-1}\left(z_{j}-y_{j}\right)^{2}-\left(\xi_{0}\right)_{n} y_{n}+i C\left(y_{n}-\left(y_{0}\right)_{n}\right)^{2}
$$

where $\Re C$ is chosen sufficiently large. In the sequel, the neighborhoods $N\left(z_{0}\right)$, $N\left(y_{0}\right)$ and the function $\Phi$ are related to the $\varphi$ of Lemma 2.1 as before.

Lemma 2.2. There is an elliptic analytic symbol $a(z, y, \lambda)$ such that the $F B I$ transformation $T$ with phase $\varphi$ and symbol a satisfies $D_{z_{n}} T=T P$ in $H_{\Phi, z_{0}}$.

That is, if $Y \subseteq Z(U)=H$ is a small neighborhood of $y_{0}$, then for $z$ in $W \subseteq C^{m}$ a small neighborhood of $z_{0}=\left(y_{0}^{\prime}-i \xi_{0}^{\prime}, 0\right)$ and $u \in \mathscr{E}^{\prime}(Y)$ we have

$$
D_{z_{n}} T u-T P u \in H_{\Phi-\varepsilon}^{\mathrm{loc}}(W)
$$

for some $\varepsilon>0$.

The symbol $a(z, y, \lambda)$ is constructed by solving the transport equations at each degree of homogeneity.

We recall now that

$$
\tilde{\gamma}(t)=(\tilde{x}(t), \tilde{\xi}(t))
$$

and

$$
\tilde{\gamma}(0)=\left(y_{0}, \xi_{0}\right)=\left(Z(x(0)), \check{Z}_{x}(x(0)) \xi_{0}\right) .
$$

Write $y_{0}=\left(y_{0}^{\prime},\left(y_{0}\right)_{n}\right)$ and $\xi_{0}=\left(\xi_{0}^{\prime},\left(\xi_{0}\right)_{n}\right)$.

We will use the equations

$$
\left\{\begin{array}{l}
\frac{\partial \varphi}{\partial z_{n}}(z, y)=p\left(y, \frac{-\partial \varphi}{\partial y}(z, y)\right) \\
\frac{\partial \varphi}{\partial y}\left(z_{0}, y_{0}\right)=-\xi^{0}
\end{array}\right.
$$

to prove that $\tilde{\xi}(t)=-\frac{\partial \varphi}{\partial y}\left(y_{0}^{\prime}-i \xi_{0}^{\prime}, t, \tilde{x}(t)\right)$.

We recall that

$$
\left\{\begin{array}{l}
\frac{d \tilde{x}}{d t}=\frac{\partial p}{\partial \zeta}(\tilde{x}(t), \tilde{\xi}(t)) \text { and } \\
\frac{d \dot{\xi}}{d t}=\frac{-\partial p}{\partial z}(\tilde{x}(t), \tilde{\xi}(t)) .
\end{array}\right.
$$

Hence

$$
\begin{aligned}
& \frac{d}{d t}\left[\frac{\partial \varphi}{\partial y}\left(y_{0}^{\prime}-i \xi_{0}^{\prime}, t, \tilde{x}(t)\right)\right] \\
& \quad=\varphi_{y z_{n}}\left(y_{0}^{\prime}-i \xi_{0}^{\prime}, t, \tilde{x}(t)\right)+\varphi_{y y}\left(y_{0}^{\prime}-i \xi_{0}, t, \tilde{x}(t)\right) \frac{d \tilde{x}}{d t} \\
& \quad=\varphi_{y z_{n}}\left(y_{0}^{\prime}-i \xi_{0}^{\prime}, t, \tilde{x}(t)\right)+\varphi_{y y}\left(y_{0}^{\prime}-i \xi_{0}^{\prime}, t, \tilde{x}(t)\right) \frac{\partial p}{\partial \zeta}(\tilde{x}(t), \tilde{\xi}(t))
\end{aligned}
$$

Now (2.4) implies that

$$
\varphi_{y z_{n}}(z, y)=\frac{\partial p}{\partial y}\left(y, \frac{-\partial \varphi}{\partial y}\right)-\frac{\partial p}{\partial \zeta}\left(y, \frac{-\partial \varphi}{\partial y}\right) \varphi_{y y}(z, y) .
$$


It follows that

$$
\begin{aligned}
& \frac{d}{d t}\left[\frac{-\partial \varphi}{\partial y}\left(y_{0}^{\prime}-i \xi_{0}^{\prime}, t, \tilde{x}(t)\right)\right] \\
& =\frac{-\partial p}{\partial y}\left(\tilde{x}(t),-\frac{\partial \varphi}{\partial y}\left(y_{0}^{\prime}-\xi_{0}^{\prime}, t, \tilde{x}(t)\right)\right) \\
& \quad+\frac{\partial p}{\partial \zeta}\left(\tilde{x}(t),-\frac{\partial \varphi}{\partial y}\left(y_{0}^{\prime}-i \xi_{0}^{\prime}, t, \tilde{x}(t)\right)\right) \varphi_{y y}\left(y_{0}^{\prime}-i \xi_{0}^{\prime}, t, \tilde{x}(t)\right) \\
& \quad-\varphi_{y y}\left(y_{0}^{\prime}-i \xi_{0}^{\prime}, t, \tilde{x}(t)\right) \frac{\partial p}{\partial \zeta}(\tilde{x}(t), \tilde{\xi}(t))
\end{aligned}
$$

But $\tilde{\xi}(t)$ also satisfies (2.5) since

$$
\begin{aligned}
\frac{d \tilde{\xi}}{d t}= & -\frac{\partial p}{\partial y}(\tilde{x}(t), \tilde{\xi}(t))+\frac{\partial p}{\partial \zeta}(\tilde{x}(t), \tilde{\xi}(t)) \varphi_{y y}\left(y_{0}^{\prime}-i \xi_{0}^{\prime}, t, \tilde{x}(t)\right) \\
& -\varphi_{y y}\left(y_{0}^{\prime}-i \xi_{0}^{\prime}, t, \tilde{x}(t)\right) \frac{\partial p}{\partial \zeta}(\tilde{x}(t), \tilde{\xi}(t)) \\
= & -\frac{\partial p}{\partial y}(\tilde{x}(t), \tilde{\xi}(t)) .
\end{aligned}
$$

Moreover, by $2.4, \frac{-\partial p}{\partial y}\left(y_{0}^{\prime}-i \xi_{0}^{\prime}, 0, y_{0}\right)=\xi_{0}=\tilde{\xi}(0)$.

We conclude that

$$
\tilde{\xi}(t)=\frac{-\partial \varphi}{\partial y}\left(y_{0}^{\prime}-i \xi_{0}^{\prime}, t, \tilde{x}(t)\right)
$$

For $t \in[0,1]$, let

$$
z(t)=z_{0}+\left(0^{\prime}, t\right)=\left(y_{0}^{\prime}-i \xi_{0}^{\prime}, t\right) \in C^{n-1} \times R .
$$

We now recall that for $z$ near $z_{0}, y(z)$ is the unique point in $N\left(y_{0}\right) \subseteq H$ such that

$$
y\left(z_{0}\right)=y_{0} \quad \text { and } \quad \frac{-\partial \varphi}{\partial y}(z, y(z)) \in\left(\Lambda_{H}\right)_{y(z)} .
$$

But by $(2.6), \tilde{\xi}(t)=\frac{-\partial \varphi}{\partial y}(z(t), \tilde{x}(t))$ and since the forms $\frac{2}{i} \partial h_{1}, \ldots, \frac{2}{i} \partial h_{n}$ are real on $H=Z(U)$ and span all of $T^{*} H$, we know that

$$
\tilde{\xi}(t)=\check{Z}_{x}(x(t)) \xi(t) \in\left(\Lambda_{H}\right)_{\tilde{x}(t)} .
$$

It therefore follows that

$$
y(z(t))=\tilde{x}(t)
$$

In our previous notation,

$$
\eta(z(t))=\frac{-\partial \varphi}{\partial y}(z(t), y(z(t)))=\frac{-\partial \varphi}{\partial y}(z(t), \tilde{x}(t))=\tilde{\xi}(t) .
$$

Thus

$$
(\tilde{x}(t), \tilde{\xi}(t))=(y(z(t)), \eta(z(t))) .
$$

Since $W F_{h a}(P u) \cap \gamma=\varnothing$ and $\gamma$ is compact, (2.7) and Proposition (2.1) tell us that

$$
T(P u) \in H_{\Phi-\varepsilon_{0}}^{\text {loc }}(N)
$$


for some $\varepsilon_{0}>0$ and a neighborhood $N$ of $\{z(t)=0 \leq t \leq 1\}$ in $C^{m}$. If $W$ is chosen as in Lemma 2.2, then

$$
D_{z_{n}} T u \in H_{\Phi-\varepsilon_{0}}^{\text {loc }}(N \cap W) .
$$

This may require a modification of $\varepsilon_{0}$.

Now $z(0)=z_{0} \in N \cap W$. Therefore, $\exists t_{1}>0$ such that $N \cap W$ is a neighborhood of $\left\{z(t): 0 \leq t \leq t_{1}\right\}$. It is crucial to note that the size of $t_{1}$ is independent of the distribution $u$.

If now $K$ is a compact neighborhood of $\left\{z(t): 0 \leq t \leq t_{1}\right\}$, then $\exists c>0$ such that

$$
\left|D_{z_{n}} T u(z, \lambda)\right| \leq c e^{\lambda\left(\Phi(z)-\frac{\varepsilon_{0}}{2}\right)} \quad \forall z \in K \text { and } \lambda \geq 1 .
$$

If $\left(y_{0}, \xi_{0}\right)=(y(z(0)), \eta(z(0))) \notin W F_{h a} u$, we know that, after modifying $c$ and $\varepsilon_{0}$,

$$
|T u(z, \lambda)| \leq c e^{\lambda\left(\Phi(z)-\frac{\varepsilon_{0}}{2}\right)} \quad \forall \lambda \geq 1 \text { and } \forall z \text { near } z_{0} .
$$

From $(2.7),,(2.8)$ and $(2.9)$, it follows that

$$
W F_{h a}(u) \cap\left\{(y(t), \xi(t)): 0 \leq t \leq t_{1}\right\}=\varnothing .
$$

\section{REFERENCES}

1. M. S. Baouendi, C. H. Chang and F. Treves, Microlocal hypo-analyticity and extensions of CR functions, J. Differential Geometry 18 (1983), 331-391.

2. S. Berhanu, Hypo-analytic pseudodifferential operators, Proc. Amer. Math. Soc. 105 (1989), 582-588.

3. _ Propagation of hypo-analyticity along bicharacteristics, Pacific J. Math 138 (1989), 221-232.

4. N. Hanges, Propagation of analyticity along real bicharacteristics, Duke Math. J. 49 (1981), 269-277.

5. L. Hörmander, An introduction to complex analysis in several variables, Van Nostrand, Princeton, N.J., 1966.

6. J. Sjöstrand, Singularités analytiques microlocales, Astérisque 95 (1982).

7. _ The FBI transform for CR submanifolds of $C^{N}$, (preprint).

8. F. Treves, Introduction to pseudodifferential and Fourier integral operators, Volume I, Plenum Press, 1980.

Department of Mathematics, Temple University, Philadelphia, Pennsylvannia 19122 